Original

\title{
Cáncer colo-rectal multicéntrico (CCRM) en el área sanitaria de León
}

\author{
A. Muela Molinero, F. Jorquera Plaza ${ }^{1}$, T. Ribas Ariño ${ }^{2}$, R. Malagón Rojo ${ }^{3}$ A. Morán Blanco, \\ A. L. Martínez Nuñez, J. Guerra Laso, J. A. Santos Calderón
}

\section{Resumen}

Objetivos: Analizar las características de los pacientes diagnosticados de CCRM en el área sanitaria de León, el impacto sobre la supervivencia y las posibles variables pronósticas.

Material y métodos: Utilizando los datos del Registro de Tumores del Hospital de León se han analizado las características de aquellos pacientes con tumores colo-rectales diagnosticados entre 1993 y 2002, clasificándolos dos grupos: el primero constituido por 74 pacientes diagnosticados de CCRM y el segundo formado por 1342 pacientes con tumores únicos diagnosticados en el bienio 1996-1997.

Resultados: La frecuencia de CCRM fue del 2,7\%. La edad de los pacientes con CCRM fue 2 años mayor con respecto a la de los pacientes con neoplasias únicas, con una elevada proporción $(38 \%)$ de antecedentes familiares oncológicos en familiares de primer grado. No se observaron diferencias significativas en cuanto a la localización y el estadio del primer tumor colo-rectal con respecto al grupo de tumores únicos. La supervivencia de los pacientes con CCRM metacrónico fue netamente inferior, posiblemente en relación con un mayor número de segundas neoplasias diagnosticadas en estadios tempranos en los pacientes con CCRM sincrónico. Las variables que influyeron en el pronóstico fueron la edad, el sexo, y el padecimiento de un CCRM sincrónico o metacrónico.

Conclusiones: El CCRM es una entidad frecuente en nuestro medio. La tasa de antecedentes familiares oncológicos en estos pacientes es muy elevada. No se encontraron diferencias significativas en cuanto al pronóstico de estos pacientes respecto al de los pacientes con neoplasias únicas.

\section{Palabras clave:}

Neoplasias múltiples. Sincrónicas. Metacrónicas. Colon. Recto. Supervivencia. Oncología. Cáncer.

Oncología, 2006; 29 (8):329-337 


\section{Summary}

Purpose: We analyze the characteristics of patients with multicentric colo-rectal cancer (MCRC) in the health district of León, the impact on survival, and the prognostic variables.

Material and methods: : Using the data from the Hospital de León Tumour Registry, we analyzed the characteristics of all the patients diagnosed of having colo-rectal tumours between 1993 and 2002. The patients were classified in two groups: one of them composed by 74 patients with MCRC, and the other formed by 1342 patients diagnosed in 1996 and 1997 of having single colo-rectal tumours.

Results: The observed frequency of MCRC corresponded to 2.7 percent of the whole cases. The age of the MCRC patients was 2 years higher than that of the patients with single neoplasms, and showed a higher familial oncologic history, that was present in 38 percent of the cases. No differences were observed for location and stage of the first appeared colo-rectal neoplasm. The survival of the patients developing metachronous MCRC was lower than for those with synchronous disease, possibly due to the higher number of the second neoplasms appearing in these patients at early stages. Prognostic values were age, sex, and metachronous or synchronous type of disease.

Conclusions: MCRC is a frequent entity. The frequency of familial oncologic history is very high. No significant differences were seen in relation to prognosis between single and multiple colorectal cancer.

Key words: Multiple neoplasms. Synchronous. Metachronous. Colon. Rectum. Survival. Prognosis. Cancer. Oncology.

\section{Introducción}

El fenómeno de las neoplasias primarias malignas múltiples (NPMM) ha pasado en las últimas décadas de ser considerado un capricho aislado de la naturaleza a establecerse como una realidad oncológica prevalente y de elevada frecuencia ${ }^{1}$. Desde 1970 se ha producido un progresivo incremento de la incidencia de NPMM en la población ${ }^{2}$, lo que ha llevado a que esta entidad haya cobrado gran importancia, al convertirse en una de las complicaciones tardías más frecuentes a las que se enfrentan los enfermos oncológicos ${ }^{3}$. Este aumento de incidencia parece relacionarse con diversos factores como la creación de los diferentes registros de tumores que han permitido un mejor control y seguimiento de los pacientes, el aumento en la longevidad de la población en general $1^{4}$, los importantes avances en las pruebas diagnósticas que han permitido la detección precoz de neoplasias en muchos casos asintomáticas $^{5,6}$, las mejoras en el campo de la oncología, tanto en el terreno de la investigación básica como en el de sus aplicaciones clínicas, lo que ha permitido incrementar la tasa de curaciones y prolongar considerablemente la supervivencia de la mayoría de los pacientes ${ }^{7}$ y el aumento de agentes carcinógenos en nuestro medio, así como el potencial efecto oncogénico de los tratamientos recibidos para la primera neoplasia ${ }^{8}$.

El estudio de las NPMM ha permitido obtener información acerca del mecanismo de acción de los agentes carcinógenos y de la presencia de determinadas alteraciones genéticas ${ }^{8}$. Sin embargo, la existencia de una enorme información, en muchos casos contradictoria, acerca de los pacientes con NPMM, así como las dificultades en la misma definición de NPMM han impedido extraer conclusiones que sirvan de ayuda al clínico en la práctica diaria ${ }^{1-4}$.

El cáncer colo-rectal es la segunda causa de muerte por cáncer en nuestro medio y la neoplasia que más frecuentemente afecta a hombres y mujeres globalmente ${ }^{9}$. El cáncer colo-rectal múltiple (CCRM) supone en muchos estudios la asociación más frecuentemente diagnosticada en los pacientes 
con NPMM, con una incidencia variable de entre el 2 y el $9 \% 10,11$, llegándose a considerar que hasta un $25 \%$ de los pacientes que sobreviven a una neoplasia colo-rectal desarrollará a lo largo del tiempo una segunda neoplasia multicéntrica ${ }^{11}$.

El presente estudio tiene como objetivos describir las características de los pacientes con CCRM en nuestra área sanitaria, incluidos en el Registro de Tumores de nuestro centro durante su primera década de funcionamiento, analizando las características de los pacientes con neoplasias sincrónicas y metacrónicas, así como analizar el impacto sobre la supervivencia del padecimiento de un CCRM.

\section{Material y métodos}

El Registro de Tumores del Hospital de León recoge información sobre todos los casos incidentes en el área sanitaria de León desde 1993, cuya población es de 366.910 personas, de las que 331.635 son atendidas por la Sanidad de Castilla y León (SACYL). Nuestro Registro de Tumores sigue las reglas de codificación para neoplasias múltiples de la IARC ${ }^{12}$. Para este análisis se incluyeron todos los pacientes diagnosticados de una neoplasia colo-rectal entre los años 1993 y 2002, que compone la primera década desde la puesta en marcha de este Registro de Tumores.

Se seleccionaron retrospectivamente aquellos pacientes diagnosticados de neoplasias colo-rectales durante el período de estudio, que fueron diagnosticados de una segunda neoplasia colo-rectal durante el período de seguimiento, que concluyó el 31 de diciembre de 2004. Se descartaron aquellos pacientes diagnosticados de alguna neoplasia maligna previamente al período de estudio. Se aplicaron los criterios descritos por Chen y cols ${ }^{13}$ según los cuales: 1) ambas neoplasias debían presentar histología maligna; 2) debía existir una separación de al menos $2 \mathrm{~cm}$ de pared sana entre las neoplasias y 3) debía descartarse que una fuera metástasis de la otra. Se consideraron sincrónicas las neoplasias diagnosticadas con intervalo igual o menor a seis meses, y metacrónicas cuando dicho intervalo era superior a seis meses.

Se recogieron las siguientes variables: la edad, el sexo, el consumo de alcohol y tabaco, los antecedentes oncológicos en familiares de primer grado, el medio de vida (urbano o rural), la fecha de diagnós- tico, la localización y el tipo histológico (según la CIE-O), el estadio (según la clasificación de Dukes), el tipo de tratamiento recibido para cada neoplasia, y la causa y fecha de éxitus, en caso de producirse éste. Las neoplasias localizadas desde ciego hasta el segmento medio del colon transverso fueron incluidas como neoplasias de colon izquierdo, mientras que aquellas localizadas entre el colon transverso y el sigma fueron incluidas como neoplasias de colon derecho. El resto se clasificaron como recto-sigmoideas.

Para el seguimiento de los pacientes el personal del Registro de Tumores revisó los historiales clínicos de cada paciente en consulta y los sucesivos ingresos en nuestro centro. Además se contactó con el paciente o un familiar directo para descartar ingresos o el fallecimiento del paciente en otro centro o en su domicilio

Para la segunda parte del estudio se seleccionaron de la base de datos del Registro de Tumores todos aquellos pacientes diagnosticados de neoplasias colo-rectales en el bienio 1996-1997, que no fueron diagnosticados de una segunda neoplasia durante el período de seguimiento. Se decidió escoger este intervalo debido a que se encontraba aproximadamente en la mitad del período de estudio, garantizándose un seguimiento mínimo de 5 años para la evaluación de la supervivencia de los pacientes. Se recogieron las siguientes variables: la edad, el sexo, el consumo de alcohol y tabaco, la fecha de diagnóstico, la localización, el tipo histológico de la neoplasia (según la CIE-9) y la fecha de fallecimiento en caso de producirse éste.

Para el análisis estadístico se utilizó el programa SPSS v11.0. Los resultados de las variables cuantitativas se expresan como mediana o media \pm desviación estándar. Para la comparación de las variables cualitativas entre los grupos definidos, se ha utilizado el test de la $\chi^{2}$ o el test exacto de Fisher cuando fue necesario, y para las variables cuantitativas se ha empleado la prueba de la t de Student. Se ha considerado significativa una $\mathrm{p}<0,05$. Para el cálculo de la supervivencia actuarial se ha empleado el método de Kaplan-Meier y, para la comparación de curvas de supervivencia el de los rangos logarítmicos. Posteriormente se ha realizado un análisis multivariante, mediante regresión de Cox, considerándose únicamente aquellas variables significativas, descartándose la presencia de colinealidad. 


\section{Resultados}

\section{Características de los pacientes con CCRM}

Durante el período de estudio se diagnosticaron un total de 2655 pacientes con neoplasias colo-rectales, de los que 1605 fueron varones y 1050 mujeres, siendo la ratio varón:mujer de 1,52:1. Se diagnosticaron 74 pacientes $(2,8 \%)$ con CCRM a lo largo del seguimiento. Un total de 43 pacientes $(58,1 \%)$ presentaron CCRM sincrónicos, mientras que 31 pacientes $(31,9 \%)$ presentaron CCRM metacrónicos. La ratio varón:mujer fue de 1,96:1.

La edad media de estos pacientes fue de $72 \pm 10$ años (44-89) y la mediana de 73 años. El 51,3\% de los pacientes eran o habían sido fumadores, mientras que un 43,2\% bebían de forma diaria al menos 20 gr/día de etanol (Tabla I). El 37,8\% de los pacientes tenían antecedentes oncológicos en familiares de primer grado, siendo el más frecuente el antecedente colo-rectal referido en un tercio de los casos. La edad de los pacientes con CCRM sincrónico fue superior, aunque no estadísticamente significativa; además se observó una mayor proporción estadísticamente significativa de varones y fumadores en este grupo de pacientes.

\section{Características de las neoplasias colo-rectales}

Todas las neoplasias colo-rectales resultaron adenocarcinomas. La localización, el estadio y el tratamiento recibido para cada neoplasia colo-rectal se muestran en la Tabla II. Solamente dos pacientes $(2,70 \%)$ presentaron metástasis al diagnóstico del primer tumor (en ambos casos hepáticas). Todos los pacientes recibieron tratamiento quirúrgico; además en 30 casos $(40,54 \%)$ se asociaron otros tratamientos alternativos a la cirugía.

El tiempo medio transcurrido entre el diagnóstico de cada neoplasia fue de $14 \pm 9$ meses $(0-62)$ y la mediana de 15 meses. Considerando los CCRM metacrónicos, el tiempo medio fue de $34 \pm 15$ meses (8-62) y la mediana de 36 meses, mientras que en el caso de los CCRM sincrónicos, el tiempo medio fue de $0 \pm 1$ meses (0-6) y la mediana de 1 mes. Solamente el 25\% de los CCRM metacrónicas fueron diagnosticados durante los dos primeros años tras el diagnóstico del primer tumor; sin embargo a los 5 años del diagnóstico se habían detectado el 96,8\% de los casos. En el momento del diagnóstico del segundo tumor colo-rectal 7 pacientes $(9,46 \%)$ presentaban metástasis a distancia, de los que 5 correspondieron a metástasis hepáticas y 2 óseas.

\section{Análisis de la supervivencia de los pacientes con CCRM}

Durante el período comprendido entre 1993 y 1998, se diagnosticaron 1342 pacientes con neoplasias colo-rectales únicas. No se encontraron diferencias significativas al comparar la edad, el sexo o la localización de cada neoplasia con respecto al grupo de pacientes con CCRM (Tabla III). La supervivencia de cada grupo se muestra en la Tabla IV. Al término del período de seguimiento habían fallecido en el grupo de neoplasias únicas 816 pacientes $(60,80 \%)$, mientras que en el grupo de CCRM habían fallecido 54 pacientes $(72,97 \%)$; esta diferencia se acercó a la significatividad estadística (p 0,06). Fallecieron por causas ajenas al tumor 241 pacientes en el grupo de neoplasias únicas $(17,96 \%)$ y 13 pacientes $(17,56 \%)$ en el grupo de CCRM, no ob-

TABLA I

Características epidemiológicas de los pacientes con NPMM

\begin{tabular}{|lccc|}
\hline Variable & $\begin{array}{c}\text { CCRM sincrónico } \\
(n=43)\end{array}$ & $\begin{array}{c}\text { CCRM metacrónico } \\
(n=31)\end{array}$ & $\begin{array}{c}\text { Significación } \\
(p)\end{array}$ \\
\hline Edad & $73 \pm 10$ & $69 \pm 11$ & $\mathrm{pNS}$ \\
Sexo (Hombre/Mujer) & $33 / 10$ & $16 / 15$ & 0,02 \\
Consumo de tabaco & $26(60,46)$ & $12(38,71)$ & 0,05 \\
Consumo de alcohol & $20(46,51)$ & $12(38,71)$ & $\mathrm{pNS}$ \\
Antecedentes oncológicos familiares & $29(67,44)$ & $15(48,39)$ & $\mathrm{pNS}$ \\
Antecedentes oncológicos colo-rectales familiares & $10(34,48)$ & $6(40)$ & $\mathrm{pNS}$ \\
Medio de vida rural & $24(55,81)$ & $10(32,26)$ & 0,03 \\
\hline
\end{tabular}


TABLA II

Estadio y tratamiento de cada neoplasia

\begin{tabular}{|c|c|c|c|}
\hline Variable & $\begin{array}{c}\text { CCRM sincrónico } \\
(n=43)\end{array}$ & $\begin{array}{c}\text { CCRM metacrónico } \\
(n=31)\end{array}$ & $\begin{array}{c}\text { Significatividad } \\
(p)\end{array}$ \\
\hline \multicolumn{4}{|c|}{ Características del primer tumor colo-rectal } \\
\hline Localización & & & $\mathrm{pNS}$ \\
\hline Colon izquierdo & $14(32,56)$ & $12(38,71)$ & \\
\hline Colon derecho & $16(37,21)$ & $8(25,81)$ & \\
\hline Recto/sigma & $13(30,23)$ & $11(35,48)$ & \\
\hline Estadio según clasificación Dukes & & & pNS \\
\hline A & $6(13,95)$ & $3(9,68)$ & \\
\hline $\mathrm{B}$ & $16(37,21)$ & $12(38,71)$ & \\
\hline $\mathrm{C}$ & $20(46,51)$ & $15(48,39)$ & \\
\hline $\mathrm{D}$ & $1(2,33)$ & $1(3,22)$ & \\
\hline \multicolumn{4}{|l|}{ Tratamiento } \\
\hline Cirugía & $43(100)$ & $31(100)$ & pNS \\
\hline Radioterapia & $2(4,65)$ & $4(12,90)$ & $\mathrm{pNS}$ \\
\hline Quimioterapia & $9(20,93)$ & $15(48,38)$ & 0,01 \\
\hline \multicolumn{4}{|c|}{ Características del segundo tumor colo-rectal } \\
\hline Localización & & & $\mathrm{pNS}$ \\
\hline Colon izquierdo & $11(25,58)$ & $10(32,26)$ & \\
\hline Colon derecho & $20(46,51)$ & $9(29,03)$ & \\
\hline Recto/sigma & $12(27,91)$ & $12(38,71)$ & \\
\hline Estadio según clasificación Dukes & & & pNS $(0,07)$ \\
\hline A & $20(46,51)$ & $6(19,35)$ & \\
\hline $\mathrm{B}$ & $13(30,23)$ & $13(41,93)$ & \\
\hline $\mathrm{C}$ & $8(18,60)$ & $7(22,58)$ & \\
\hline $\mathrm{D}$ & $2(4,65)$ & $5(16,12)$ & \\
\hline \multicolumn{4}{|l|}{ Tratamiento } \\
\hline Cirugía & $42(97,67)$ & $26(83,87)$ & 0,04 \\
\hline Radioterapia & $1(2,33)$ & $3(9,67)$ & $\mathrm{pNS}$ \\
\hline Quimioterapia & $4(9,30)$ & $3(9,67)$ & $\mathrm{pNS}$ \\
\hline Tratamiento sintomático & $1(2,33)$ & $5(16,13)$ & 0,04 \\
\hline
\end{tabular}

TABLA III

CCRM vs neoplasias colo-rectales únicas

\begin{tabular}{|c|c|c|c|}
\hline Variable & $\begin{array}{l}\text { Tumores colo-rectales únicos } \\
\qquad(n=1342)\end{array}$ & $\begin{array}{l}C C R M \\
(n=74)\end{array}$ & $\begin{array}{c}\text { Significación } \\
(p)\end{array}$ \\
\hline Edad & $70 \pm 11$ & $72 \pm 10$ & pNS \\
\hline Sexo (Hombre/Mujer) & $766 / 576$ & $49 / 25$ & pNS \\
\hline Localización del primer tumor & & & pNS \\
\hline Colon & $823(62,07)$ & $50(67,56)$ & \\
\hline Recto & $519(37,93)$ & $24(32,44)$ & \\
\hline
\end{tabular}

servando diferencias significativas en este sentido. La proporción de pacientes fallecidos por causa directamente relacionada con la neoplasia fue significativamente superior en el grupo de pacientes con CCRM sincrónico (Fig. 1).

En el análisis de supervivencia mediante Kaplan-
Meier no se encontraron diferencias estadísticamente significativas al comparar el grupo de pacientes con CCRM y aquellos con neoplasias únicas, considerando la fecha de inicio de seguimiento como la de diagnóstico del primer tumor (Fig. 2). Incluso la supervivencia de los pacientes fue ligeramente su- 
TABLA IV

Supervivencia de los pacientes

\begin{tabular}{|c|c|c|c|c|}
\hline & Exitus & 2 años & 5 años & $\begin{array}{l}\text { Supervivencia media } \\
\text { en meses (IC 95\%) }\end{array}$ \\
\hline \multicolumn{5}{|c|}{$\begin{array}{l}\text { Supervivencia desde el diagnóstico } \\
\text { del primer tumor colo-rectal }\end{array}$} \\
\hline CCRM sincrónicas & $29(67,44)$ & $53,13 \%$ & $32,74 \%$ & $53 \pm 8(37-69)$ \\
\hline CCRM metacrónicas & $24(77,42)$ & $90,32 \%$ & $40,76 \%$ & $64 \pm 7(49-78)$ \\
\hline Tumores colo-rectales únicos & $816(60,80)$ & $58,82 \%$ & $42,75 \%$ & $57 \pm 1(54-50)$ \\
\hline
\end{tabular}

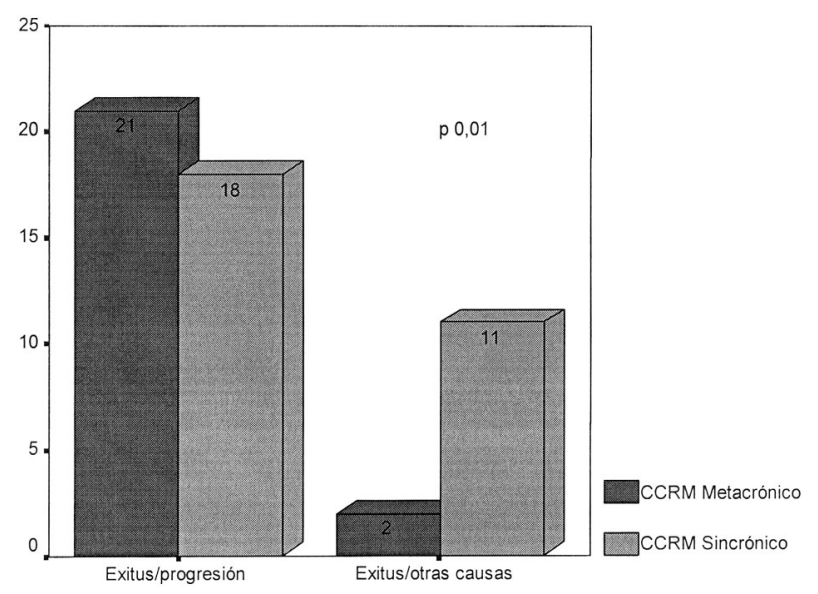

Figura 1. Causa del éxitus en los pacientes con CCRM.

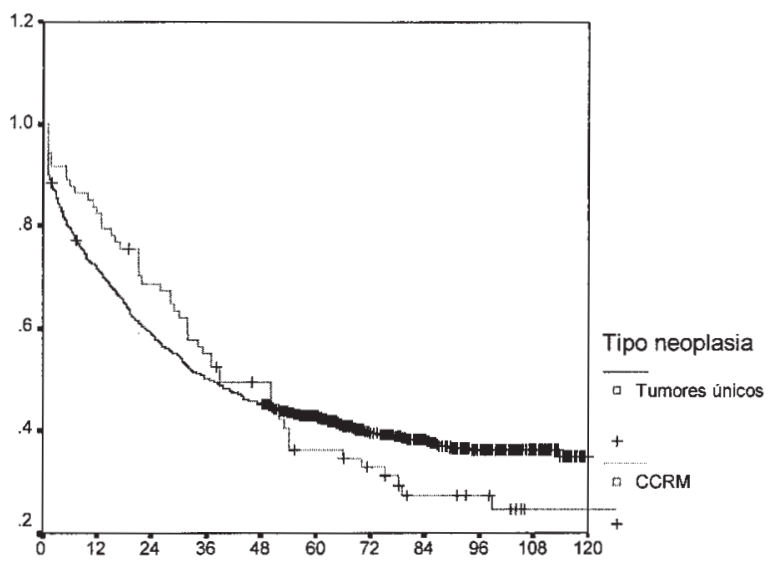

Supervivencia en meses desde el primer tumor

Figura 2. Supervivencia CCRM vs neoplasias colo-rectales únicas.

perior durante los primeros 60 meses, en gran parte por el decalaje de tiempo hasta la aparición de la segunda neoplasia colo-rectal en los pacientes con CCRM metacrónico. De igual modo, al comparar la
TABLA V

Resultados del análisis multivariante

\begin{tabular}{|lccc|}
\hline Variable & Significación & $R R$ & IC del 95\% \\
\hline Edad & 0,0001 & 1,0312 & $1,0109-1,0833$ \\
Sexo varón & 0,0309 & 2,1670 & $1,0737-4,3733$ \\
CCRM sincrónico & 0,0113 & 0,6970 & $0,5273-0,9215$ \\
$\begin{array}{l}\text { Estadio del primer } \\
\text { tumor }\end{array}$ & 0,0012 & 1,3109 & $1,0121-1,9104$ \\
$\begin{array}{l}\text { Estadio del segundo } \\
\text { tumor }\end{array}$ & 0,0410 & 1,2156 & $1,0651-1,5428$ \\
\hline
\end{tabular}

supervivencia de los pacientes con CCRM sincrónicos y metacrónicos, tomando como referencia la fecha de diagnóstico del segundo tumor, no se encontraron diferencias significativas.

Tras el análisis multivariante mediante regresión de Cox (Tabla V), las variables con significado pronóstico que resultaron estadísticamente significativas fueron: la edad, el sexo, el estadio de la cada neoplasia y el padecimiento de un CCRM sincrónico o metacrónico.

\section{Discusión}

El CCRM es la neoplasia multicéntrica más frecuente $^{10-14}$. Su frecuencia ha ido aumentando en la última década, hasta situarse en torno al 2-9\% siendo de un 1-9\% en el caso de CCRM sincrónico, y en torno al 2-6\% si se trata de CCRM metacróni$\mathrm{co}^{15-17}$. Los datos de nuestro estudio son similares a los de trabajos previos, aunque se sitúan en la parte baja de la horquilla de frecuencias, mostrando una frecuencia de CCRM del 2,78\%, siendo la frecuencia del CCRM sincrónico del 1,61\%, mientras que en el caso del CCRM metacrónico fue del 1,16\%. 
En el caso de la detección de CCRM metacrónicos se han descrito dos picos, uno durante los primeros 4-5 años y otro a partir de 10-12 años, relacionándose el primer pico con tumores sincrónicos no detectados en el primer tumor que ha evolucionado en el tiempo ${ }^{18}$. Dado que en nuestro estudio no se ha realizado un seguimiento a largo plazo de los pacientes, muy posiblemente en los próximos años se detecten más casos de CCRM metacrónicos tardíos que hagan que la frecuencia de éstos en nuestra serie, se sitúe dentro del rango habitual descrito en la literatura.

La incidencia de CCRM aumenta con la edad y por lo general aparece en pacientes de edad avanza$\mathrm{da}^{14}$. El CCRM sincrónico parece presentarse en pacientes con edades superiores a la población oncológica general ${ }^{19}$, mientras que el CCRM metacrónico se presenta en pacientes más jóvenes ${ }^{17,20}$. En nuestra serie esta afirmación se cumplió, ya que la edad de los pacientes con tumores sincrónicos fue de 73 años, mientras que la de los pacientes con tumores metacrónicos fue de 69 años y la de los pacientes con neoplasias únicas fue de 70 años, aunque no se observaron diferencias estadísticamente significativas.

Aunque muchos autores no han hallado un claro predominio del CCRM en la distribución por se$\operatorname{xos}^{13,21,22}$, los datos globales de nuestra serie siguen la tendencia de otros estudios previos ${ }^{21,23}$, al observar un predominio de la detección de CCRM en varones.

La etiopatogenía del CCRM es múltiple, habiéndose relacionado con la secuencia adenoma-carcinoma, diferentes carcinógenos externos (relacionados principalmente con un excesivo consumo de hidrocarburos refinados y grasas saturadas y una escasa ingesta de fibra, así como el consumo de alcohol) y una base hereditaria. De todos estos factores, la secuencia adenoma-carcinoma parece el más importante en la génesis del CCRM ${ }^{24}$.

Distintos trabajos han constatado una mayor frecuencia de antecedentes familiares oncológicos entre los pacientes con NPMM ${ }^{18-22}$. Nuestra serie presentó una elevada tasa de antecedentes familiares oncológicos directos $(37,84 \%)$, de los que un tercio fueron neoplasias colo-rectales. Aunque no contamos con datos similares de una población control esta tasa es con seguridad claramente superior a la de la población general. Esta elevada proporción su- giere la existencia de una base hereditaria en los pacientes con CCRM. No contamos con datos acerca de los hábitos dietéticos de los pacientes incluidos en el estudio, pero la tasa de consumo de tabaco y alcohol fue también elevada (51 y $43 \%$ respectivamente), lo que podría reflejar la importancia de los agentes externos en el desarrollo de estas neoplasias. En este sentido también podría ser muy importante el papel de la inestabilidad de los microsatélites ${ }^{19,21,24}$, aunque no está clara su relación con la neoplasia de colon hereditaria, aunque tampoco se ha encontrado una mayor frecuencia de cáncer de endometrio; desgraciadamente en nuestro trabajo se ha podido constatar dicha posibilidad, por lo que no podemos extraer conclusiones al respecto.

La localización más frecuente del CCRM sincrónico es el recto y sigma ${ }^{14,20,25}$, aunque también existen trabajos que hablan del colon derecho como principal localización y otros que hablan de una afectación universal ${ }^{22}$. Los CCRM metacrónicos parecen asentar preferentemente en el colon izquier$\mathrm{do}^{26}$. Sin embargo, en nuestro estudio la localización preferente de los CCRM fue el colon derecho y el recto/sigma, no hallando diferencias significativas en cuanto a neoplasias sincrónicas y metacrónicas.

El estadio de las primeras neoplasias suele ser similar (habitualmente Dukes B o C en el 70-80\% de los casos). Sin embargo, las segundas neoplasias metacrónicas suelen presentarse en estadios más avanzados, en relación con el estadio de las neoplasias sincrónicas, quizá debido a que no fueran diagnosticadas durante el período de estadiaje del primer tumor ${ }^{14}$. No obstante, en este punto también existen importantes controversias, ya que como señalan Fajobi y cols ${ }^{17}$, también hay otros estudios en los que el estadio del primer tumor de los pacientes con neoplasias metacrónicas, en muchos casos tiende a ser más favorable, y quizá por ello se desarrollen más neoplasias metacrónicas en este grupo de pacientes. Nuestro estudio sigue dicha tendencia, demostrando que el 70\% de los CCRM se detectan en estadio A-B de Dukes.

Muchos estudios previos coinciden en afirmar que el pronóstico de los pacientes con CCRM metacrónico es mejor ${ }^{14,19}$. En algunas ocasiones esta situación se puede explicar porque se toma como referencia la fecha de diagnóstico del primer tumor, por lo que se ignora el decalaje de intervalo libre de 
enfermedad hasta el diagnóstico del tumor metacrónico ${ }^{14-16}$. En otros casos los pacientes que posteriormente desarrollan CCRM metacrónicos suelen ser aquellos pacientes cuyo primer tumor tuvo un mejor pronóstico. Sin embargo, los datos de nuestra serie no confirman esta última hipótesis. El estadio de la primera neoplasia fue similar en todos los grupos, pero en los pacientes con CCRM sincrónicos se detectaron un mayor número de segundas neoplasias en estadios precoces, por lo que la inspección minuciosa de todo el intestino grueso en la etapa de estadiaje del primer tumor, sigue siendo la mejor arma para mejorar la supervivencia de estos pacientes. Incluso el pronóstico de los pacientes con CCRM sincrónico fue similar al de los pacientes con neoplasias únicas. Las variables que influyeron como factores pronósticos en nuestro estudio no fueron las relacionadas con la localización o el estadio del tumor, sino las relacionadas con el paciente (sexo y edad), así como el padecimiento de un CCRM sincrónico o metacrónico, independientemente del estadio.

\section{Conclusiones}

El CCRM constituye un problema relativamente frecuente en nuestro medio. La elevada frecuencia de antecedentes oncológicos familiares en estos pacientes sugiere una posible base hereditaria que actuaría como factor predisponente junto al resto de factores vinculados a la etiopatogenia de esta entidad. El pronóstico del CCRM es similar al de los pacientes con neoplasias únicas, sin encontrar diferencias entre los pacientes con neoplasias sincrónicas y metacrónicas.

Correspondencia:

Dr. A. Muela Molinero

C/ Abad de Santillán, 15 - 2 A

E-24008 León

albmuela@yahoo.es

\section{Bibliografía}

1. Cahan WG. International Workshop on multiple primary cancers. Introductory remarks. Cancer 1977; 40: 1785.

2. Watanabe S, Kodama T, Shimosato Y, Arimoto H, Sugimura T, Suemasu K, et al. Multiple primary cancers in 5,456 autopsy cases in the National Cancer Center of Japan. J Natl Cancer Inst 1984; 72: 1021-1027.

3. Feliu J. Neoplasias: dos peor que una. Med Clin (Barc) 1994; 102: 136-138.

4. Demandante CG, Troyer DA, Miles TP. Multiple primary malignant neoplasms: case report and a comprehensive review of the literature. Am J Clin Oncol 2003; 26: 79-83.

5. Doll R. Progress against cancer and epidemiologic assessment. Am J Epidemiol 1991; 134: 675-688.

6. Koyama K, Furukawa Y, Tanaka H. Multiple primary malignant neoplasms in urologic patients. Scand J Urol Nephrol 1995; 29: 483-490.

7. Shibuya H, Wakita T, Nakagawa T, Fukuda H, Yasumoto $\mathrm{M}$. The relation between an esophageal cancer and associated cancers in adyacent organs. Cancer 1995; 76: 101-105.

8. Schottenfeld D. Multiple primary cancers. En: Schottenfeld D, Fraumeni JF, eds. Cancer epidemiology and prevention. New York: Oxford University Press, 1996; 13701387.

9. Axon ATR, Boyle P, Ridell RH. Summary of a working party on the surveillance of premalignant lesions. Am J Gastroenterology 1994; 89 (Suppl): 160-168.

10. Shah IA, Alfsen GC. Multiple primary malignant tumors involving the large bowel. Dis Colon Rectum 1984; 27: 798-802.

11. Takeuchi H, Toda T, Nagasaki S, Kawano T, Minamisono Y, Maehara Y, et al. Synchronous multiple colorectal adenocarcinomas. J Surg Oncol 1997; 64: 304-307.

12. International rules for múltiple primary cancers. IARC, Lyon 2004. Int report No 2004/12.

13. Chen H-S, Sheen-Chen S-M. Synchronous and "early" metachronous colorectal adenocarcinoma. Dis Colon Rectum 2000; 43: 1093-1099.

14. Villalba F, Bernal JC, Fuster CA, Asensi J, Vázquez A, García MJ. Tumores múltiples de colon. Estudio protocolizado de 450 carcinomas colo-rectales. Rev Esp Enf Digest 1997; 89: 759-763.

15. Ekelund GR, Pihl B. Multiple carcinomas of the colon and rectum. Cancer 1974; 33: 1630-1634.

16. Cali RL, Pitsch RM, Torzón AG, Watson P, Tapia P, Blatchford GJ et al. Cumulative incidence of metachronous colorectal cancer. Dis Colon Rectum 1993; 36: 388393.

17. Fajobi O, Yiu C-Y, Sen-Gupta B, Boulos PB. Metachronous colorectal cancers. Br J Surg 1998; 85: 897-901.

18. Attiyeh FF. Guidelines for the follow-up of patients with carcinomas and adenomas of the colon and rectum. En Stearns MW Jr. ed. Neoplasms of the Colon, Rectum and Anus. New York: John Wiley and sons, 1980; 193-197. 
19. Passman MA, Pommier RF, Vetto JT. Synchronous colon primaries have the same prognosis as solitary colon cancers. Dis Colon Cancer 1996; 39: 329-334.

20. Welch JP. Multiple colorectal tumors. An appraisal of natural history and therapeutic options. Am J Surg 1981; 142: 274-280.

21. Adloff M, Arnaud JP, Bergamaschi R, et al. Synchronous carcinoma of the colon and rectum: prognostic and therapeutic implications. Am J Surg 1999; 157: 299-302.

22. Gómez Iglesias S, Cuñat E, Torregrosa S, et al. Carcinomas múltiples sincrónicos del colon y recto. Presentación de 18 casos y revisión de la literatura. Rev Esp Enf Ap Digest 1989; 76: 316-320.
23. García Gil FA, Jiménez Bernardo A, González Morán JM, Lamata Hernández J, González González M. Cirugía del cáncer de recto. Análisis retrospectivo de una serie de 123 casos. Rev Esp Enf Ap Digest 1984; 66: 385-394.

24. Rudy DR, Zdon MJ. Update on colorectal cancer [see comments]. Am Fam Physician 2000; 61: 1759-1770.

25. Kaibara N, Koga S, Sinnai D. Synchronous and metachronous malignancies of the colon and rectum in Japan with special reference to a coexisting early cancer. Cancer 1984; 54: 1870-1874.

26. Rennert G, Robinson E, Rennert HS, Neugut AI. Clinical characteristics of metachronous colorectal tumors. Int $\mathbf{J}$ Cancer 1995; 60: 743-747. 